\title{
OCULAR CHANGES OCCURRING DURING CARDIAC SURGERY UNDER PROFOUND HYPOTHERMIA AND OCCLUSION*
}

\author{
BY \\ ADRIAN LAMB \\ Perth, Western Australia
}

To carry out intra-cardiac operations under direct vision it is essential to occlude completely the cardiac inflow and thus to achieve a dry field for surgery.

Until recently this has been done in two different ways:

(1) HyPothermia.-Cooling reduces body metabolism and therefore its oxygen requirements, and when a patient is cooled from the normal $37^{\circ} \mathrm{C}$. to $30^{\circ} \mathrm{C}$. it is possible to occlude the circulation for up to 6 to 7 minutes with safety. The method is limited by the fact that at temperatures of $25^{\circ}-$ $30^{\circ} \mathrm{C}$. ventricular fibrillation or cardiac asystole is very liable to occur.

(2) Extracorporeal Circulation.-By this means the heart and lungs are bypassed completely by a pump oxygenator. This method is complex and difficult, and requires elaborate machinery and a large quantity of blood to prime the apparatus. Additional hazards are encountered when induced cardiac arrest is employed to make intra-cardiac surgery easier.

In an endeavour to overcome these disadvantages, Drew and Anderson (1959) employed deep hypothermia down to $15^{\circ} \mathrm{C}$., at which temperature they were able to occlude the circulation completely for up to 45 minutes without neurological damage. An extracorporeal circuit was employed to cool the blood and to assist the circulation when it failed as hypothermia deepened.

The method of Drew and Anderson (1959) has been modified by Simpson, Gibson, and Bloomfield (1960), who achieve profound hypothermia in their patients by using a comparatively low flow bypass through a pumpoxygenator-heat-exchanger unit. Adequate circulation is maintained by the pump-oxygenator while cooling and re-warming are carried out, and complete circulatory arrest for up to 43 minutes has been safely achieved.

The physiological responses of the body to such profound hypothermia and prolonged circulatory arrest are being closely investigated, but no work has so far been published on the ocular changes which occur. These ocular observations should be considered in relation to the wider physiological field

* Received for publication July 5, 1960. 
and may therefore be of assistance to those engaged in such work, but they may in themselves be of special interest to ophthalmologists.

The ocular changes to be described are taken from our first fifteen patients to be operated upon for cardiac defects under profound hypothermia and occlusion, backed by observations and experiments carried out on dogs. The report is put forward at this early stage, first because certain physiological patterns of response have already become apparent, and secondly because the recording of these patterns may be of use to other workers.

\section{General TeChNiQue}

Before describing and discussing the ocular changes it is necessary to delineate the major steps involved in the general procedure, in order that these changes can be related and assessed:

(1) Premedications.-Atropine, Pethedine, Phenergan.

(2) Anaesthesia.-Induction is by intravenous pentothal and relaxants, followed by maintenance on nitrous oxide and oxygen.

(3) During Anaesthesia.-The chest is opened, venous and arterial cannulae are placed, and the patient is made ready for circulatory bypass cooling. This period of anaesthesia may last up to 2 hours and its influence on the pupillary reactions and intra-ocular pressure is reviewed later in this paper.

(4) Bypass Cooling.-When preparations are complete, a mechanical pump is turned on which circulates the blood through an oxygenator and cooling device, resulting in a gradual lowering of the body temperature to from $18^{\circ}$ to $15^{\circ} \mathrm{C}$. pharyngeal $\left(12^{\circ}\right.$ to $10^{\circ} \mathrm{C}$. mediastinal). This process takes from 15 to 20 minutes.

(5) Occlusion.-The pump is then stopped and the patients remain in complete circulatory arrest for 12 to 30 minutes (in one case for 43 minutes), during which period the cardiac surgery is performed. Anaesthesia and ventilation of the lungs are suspended until bypass re-warming is started.

(6) Bypass Re-warming.-When the cardiac surgery is completed the pump is restarted. Using a warming device the body temperature gradually rises to reach normal in about 30 minutes.

(7) The Heart.-During cooling, the heart beat becomes ineffective and often ventricular fibrillation occurs. At very low temperatures the heart is practically motionless-providing ideal conditions for surgery. Ventricular fibrillation may again occur on re-warming but electrical defibrillation is easily carried out when the temperature reaches about $30^{\circ} \mathrm{C}$.

\section{OCULAR OBSERVATIONS}

In the following description, pharyngeal temperatures are used, as the recording thermometer is readily accessible and probably represents a level nearer to that of the eye than the much lower intra-thoracic temperature. Where indicated the thoracic temperature is noted in brackets. 
The following ocular changes have been observed:

The PuPIL (Fig. 1).

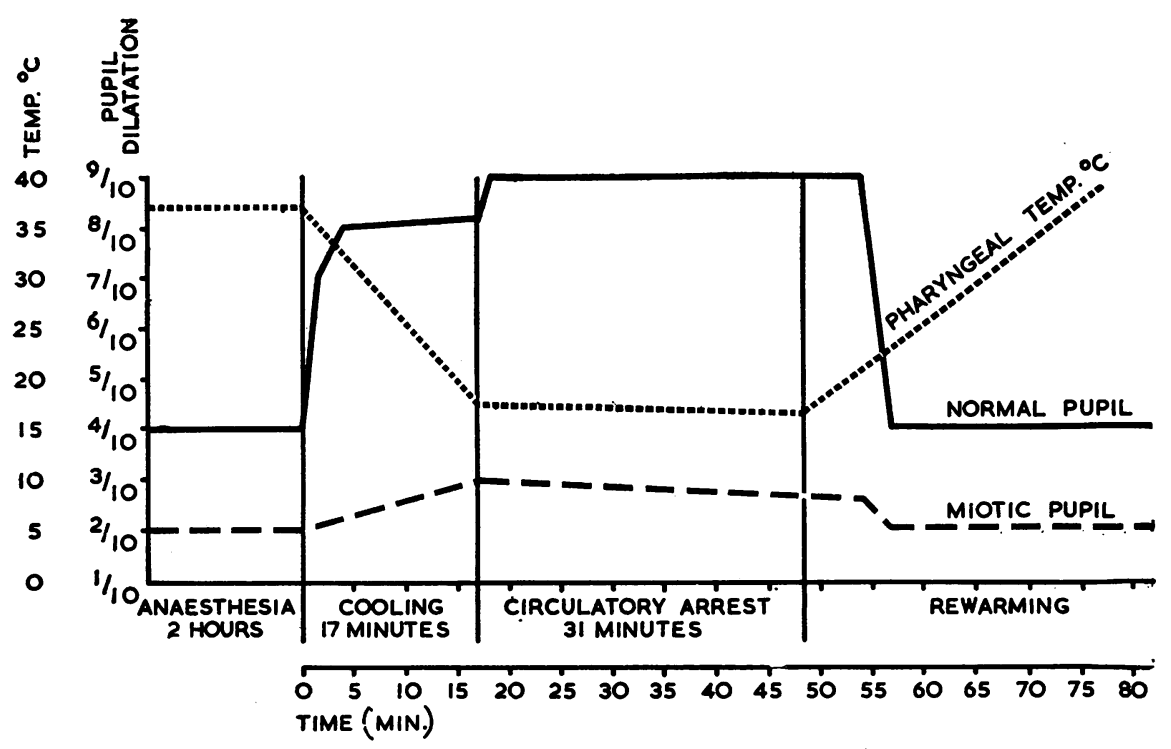

FIG. 1.-Case 4, recording of the behaviour of the normal pupil and of a pupil under miosis in relation to the body temperature and a period of occlusion.

The method of describing maximum mydriasis as $10 / 10$ dilatation, the fraction decreasing to $1 / 10$ for maximum miosis, has been used throughout.

Anaesthesia.-During this period the pupil has behaved according to accepted expectations showing an average of $4 / 10$ dilatation and being unaffected by the premedication.

Bypass Cooling.-At the onset of bypass cooling the pupil remains static for 45 seconds and then rapidly dilates. It reaches $7 / 10$ as the temperature falls until, at a pharyngeal reading of $17^{\circ} \mathrm{C} .\left(10^{\circ} \mathrm{C}\right.$.), the pupil is at $8 / 10$ dilatation.

Occlusion.-At this temperature the bypass pump circulation is cut off and total circulatory arrest occurs. Immediately, there is a rapid dilatation of the pupil from $8 / 10$ to $9 / 10$ and this dilatation continues throughout the whole period of occlusion. During this period there is some fall in the temperature, usually of about $1^{\circ}$, but the pupil does not alter.

Bypass Re-warming.-The process of warming and oxygenating the patient commences as soon as the bypass circulation pump is restarted. For 
7 minutes the pupil is unaffected. During this period there is restoration of a mean blood pressure of from 50 to $70 \mathrm{~mm}$. $\mathrm{Hg}$, recovery of a degree of intra-ocular pressure, and a rise in pharyngeal temperature to about $20^{\circ} \mathrm{C}$. At 7 minutes the pupil rapidly contracts during a period of 90 seconds to normal. This occurs fairly constantly at a pharyngeal temperature of $22^{\circ} \mathrm{C}$. At about the same time the intra-ocular pressure is found to return to normal. Following the return to normal of the pupil at this temperature, it remains constant thereafter in spite of a rise in the body temperature which continues until the normal level is reached. In some cases, pupil reactions to light are found to occur when the body temperature rises to about $30^{\circ} \mathrm{C}$., but this varies according to the depth of anaesthesia present at the moment.

Unusual Pupil Reactions.-It has been observed on two occasions that, when the bypass pump is cut off after re-warming and the patient is returned to his normal circulation, there is a transitory rise in the size of the pupil. This rise lasts for only about 30 seconds whereupon the pupil reverts to normal.

Defibrillation.-When defibrillation is electrically induced the pupil shows a very slight transient enlargement starting one second after the cardiac electrical stimulus. In dogs this dilatation has been much more marked and a summation effect has been observed where repeated electrical applications have been necessary.

Autonomic Nervous System.-It has been assumed that, during the occlusion period of this operation, the autonomic nervous system is completely paralysed. For this reason, pupils have been subjected to the influence of pilocarpine and observations have been followed accordingly.

Miotic Pupil.-In general, the pupil under miosis remains so throughout anaesthesia. As soon as bypass cooling is started, the miotic pupil increases slightly in size but to a very much less extent than the normal. At the onset of occlusion, there is a sharp rise in the dilatation of the pupil, which thereafter decreases in diameter during occlusion. When the bypass is started again in the re-warming process, the miotic pupil rapidly descends to its original miotic state, this process occurring within 7 to 8 minutes. Having reached its miotic state, the pupil remains constant throughout the remainder of the bypass re-warming period and after the bypass re-warming pump has been stopped and normal circulation is re-established. It has been found that the behaviour of the pupil under the influence of miosis varies directly with the degree of miosis and the intensity of the miotic stimulus. Thus the miotic pupil induced before anaesthesia will dilate to some degree during occlusion ( 2 hours later), whereas little or no dilatation occurs if the pilocarpine is instilled only half an hour before occlusion. 
The Intra-ocular Pressure

Anaesthetic Influences.-Anaesthesia normally produces a reduction in intra-ocular pressure. This is further affected by the use of relaxants and under ordinary anaesthetic conditions the intra-ocular pressure may be expected to fall during the induction and maintenance of anaesthesia.

Factors Influencing Intra-ocular Pressure.-The intra-ocular pressure of the human eye has formed the subject of a great deal of investigation for many years and the true nature of its maintenance is not yet fully understood. There are many factors involved, including the arterial blood pressure, the facility of outflow from the eye, the osmotic pressures, and various biochemical reactions which can occur in the eye. The subject is of immense importance in relation to glaucoma. It is thought that the blood pressure in the capillaries, particularly those involving the ciliary body, is of major importance, and it is suggested from this study that the intraocular blood volume may also be a factor requiring consideration.

In the present series, this pressure has been studied carefully and the following observations have been made (Fig. 2).

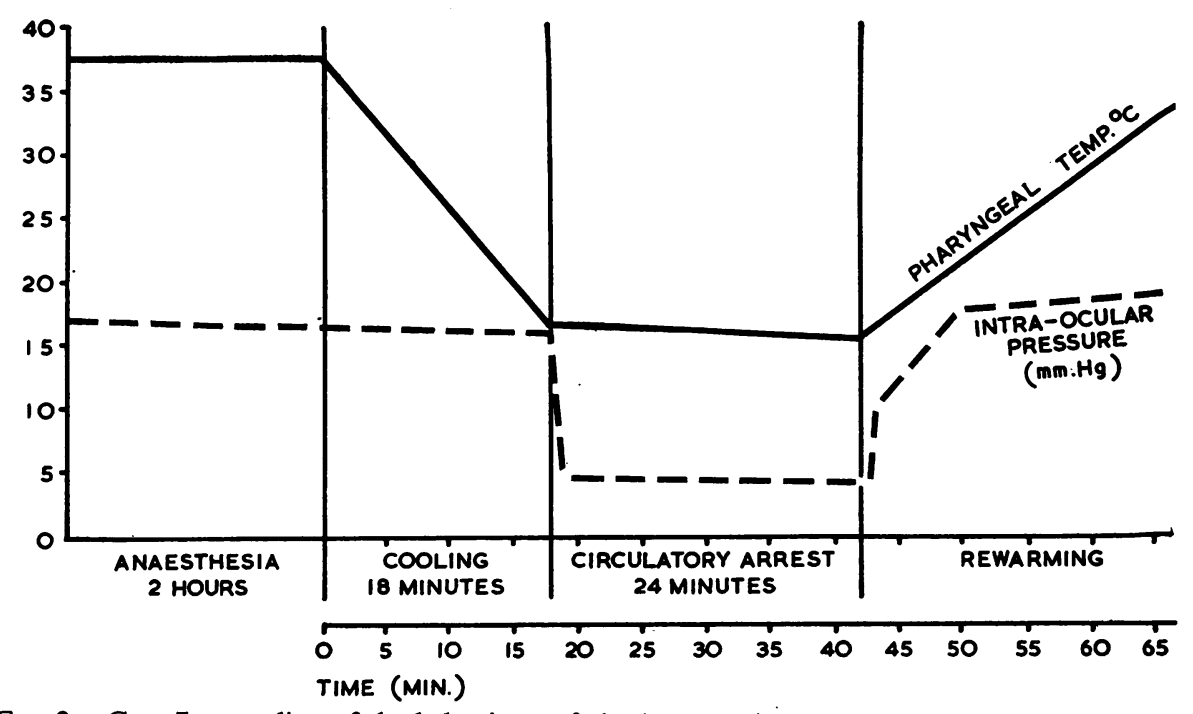

FIG. 2.-Case 7, recording of the behaviour of the intra-ocular pressure in relation to the body temperature and a period of circulatory arrest.

Anaesthesia.-During anaesthesia, the intra-ocular pressure in all patients has been found to be lower than the normal, varying between 18 and $16 \mathrm{~mm}$. Hg. This conforms very well with our studies previously made in lytic cocktail anaesthesia. During anaesthesia there has been a slow fall of about $2 \mathrm{~mm}$. $\mathrm{Hg}$. 
Bypass Cooling.-During bypass cooling the intra-ocular pressure has been fairly constant, showing only a very slight fall.

Occlusion.-At the onset of occlusion, the intra-ocular pressure falls very rapidly to $4 \mathrm{~mm}$. $\mathrm{Hg}$, which is about the lowest recordable pressure. This has been accurately timed to occur within 16 seconds of the onset of occlusion. During the occlusion, the intra-ocular pressure has slightly declined.

Bypass Re-warming.-The critical period of interest concerning intraocular pressure is at the onset of bypass re-warming where the arterial circulation is restored by artificial means. At this point the intra-ocular pressure is almost nil and it is felt that the sudden onset of a positive intraocular arterial pressure could exert considerable strain on the retinal arterioles and capillaries which are not supported by their normal ocular tension.

All cases have been reviewed from this point of view and the most recent cases have been examined on a 5-second interval basis (Fig. 3). It appears that there is no response to the return of circulation for about 15 seconds, during which period the intra-ocular pressure remains almost nil. From 15 seconds to one minute, the intra-ocular pressure recovers to about half its normal level. From this period onwards there is a slow rise to normal over a period of 9 minutes. It should be pointed out that there are certain

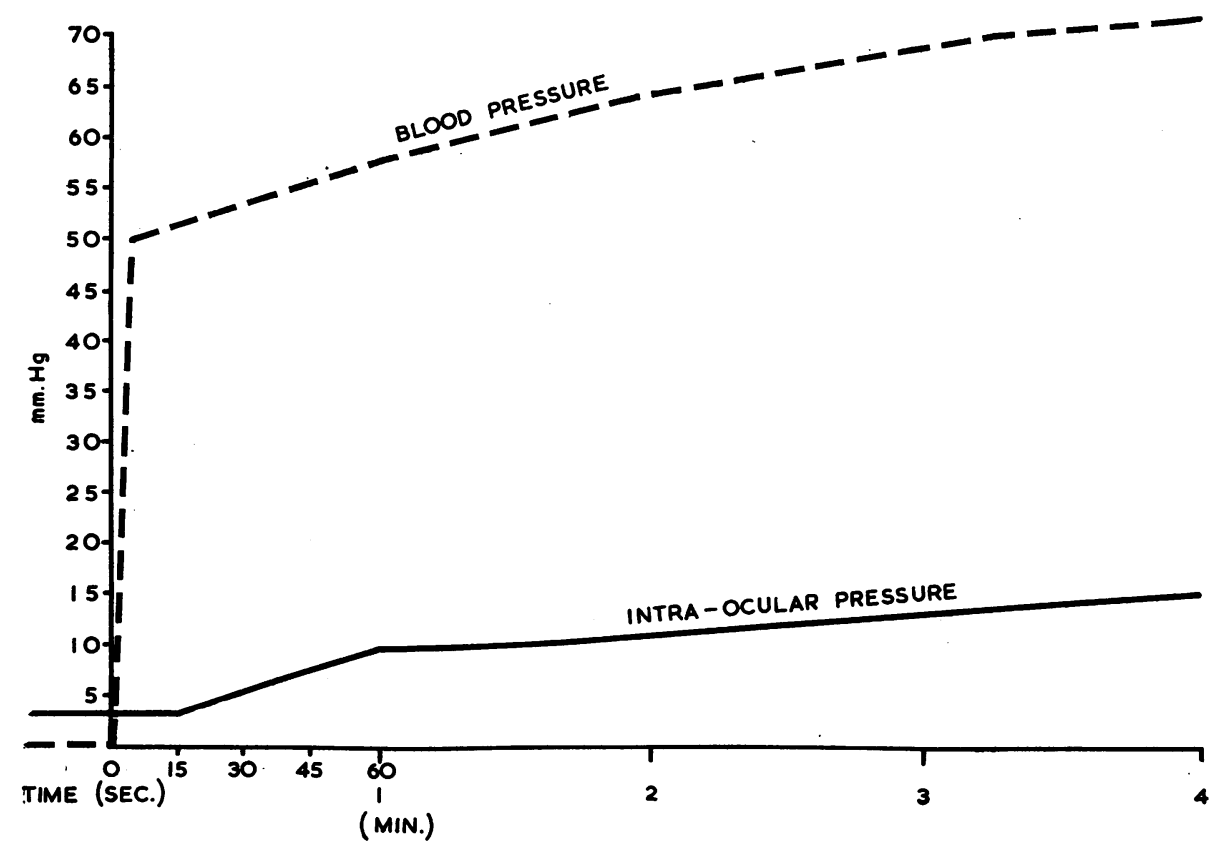

FIG. 3.- Relative recovery rate of the intra-ocular pressure and the blood pressure after the restoration of circulation. 
clinical fallacies in the estimation of this intra-ocular pressure. The repeated weight of the tonometer on the eye in itself tends to reduce the intra-ocular pressure, and this factor must be borne in mind when repeated readings are taken at 5-second intervals. In several cases, however, the eyes were used alternately and it would appear that this record may be reasonably accurate.

During the period of re-warming, the intra-ocular pressure, having returned to $16-18 \mathrm{~mm}$. $\mathrm{Hg}$, remains at that level and is unaltered when the normal circulation is restored. At this time, of course, the intra-ocular pressure is again under the influence of anaesthesia.

In dogs the insertion of cannulae into the anterior chamber has shown some recovery of intra-ocular pressure at 7 seconds with a rapid increase up to 35 seconds. Thereafter the recovery has been slow.

Blood Pressure.-During occlusion the blood pressure is of course nil. Within 5 seconds of circulatory restoration the mean radial artery pressure reaches $50 \mathrm{~mm}$. $\mathrm{Hg}$, rising in about 2 minutes to $70 \mathrm{~mm}$. $\mathrm{Hg}$, and slowly thereafter to normal in 9 minutes (Fig. 3).

\section{Fundus Changes}

The fundus changes in occlusion have not previously been described. The following observations are based on all our cases which have been examined throughout the whole of the occlusion period and are supported by similar findings in dogs (Figs 4, 5, and 6). Colour photographs, using a portable fundus camera, have been taken during the period of circulatory arrest. At the onset of occlusion, there is an immediate pallor of the optic disc which is due to the collapse of the small disc capillaries.

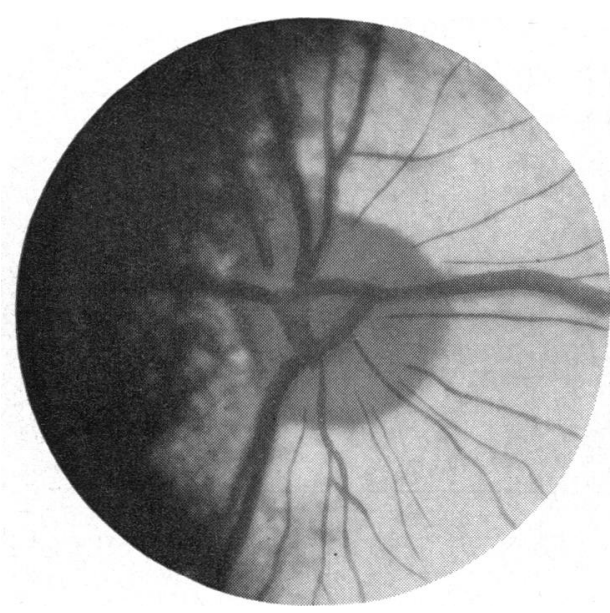

FIG. 4.-Normal dog fundus.

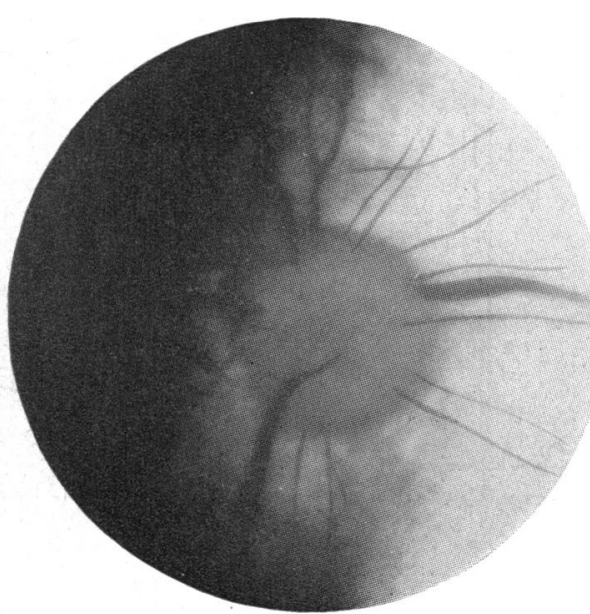

Fig. 5.-Dog fundus 15 seconds after total occlusion of circulation at $15^{\circ} \mathrm{C}$. 


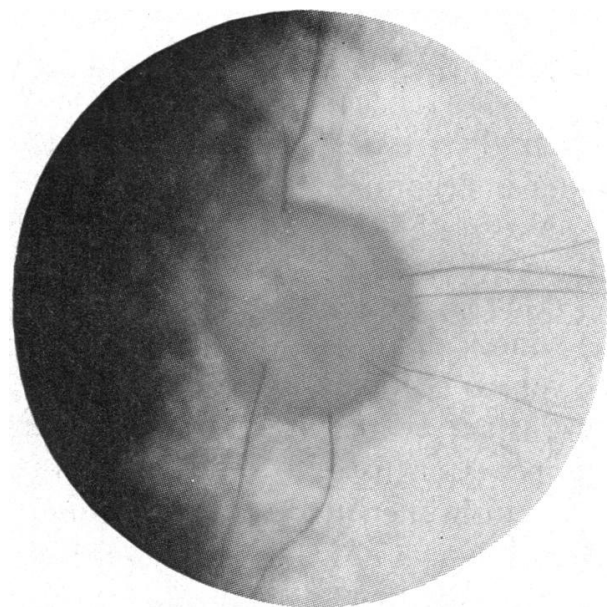

Fig. 6. -Dog fundus after 15 minutes of total occlusion at $15^{\circ} \mathrm{C}$.

Collapse of Arteries and Veins.-Within a few seconds, the arterioles and veins of the retina become flattened.

Arterial and Venous Colour.-Within 3 to 4 minutes of occlusion the arteries and veins both become the same colour and there is obvious irregularity of the venous wall.

Interruption of Arteries and Veins at the Disc.-After from 6 to 7 minutes the vessels leaving the disc become completely obliterated and are invisible at this point. This process becomes more noticeable later in the occlusion period.

Fragmentation of the Blood Column.-This is the next event; it begins early towards the disc margin and extends throughout the fundus. It tends to become severe within about 12 minutes of occlusion.

Loss of Fundal Colour.-Towards the 15th minute of occlusion, loss of the normal red reflex of the fundus becomes evident.

Choroidal Pattern Visible.-Shortly after the fundal colour is lost, the choroidal vessels become visible and some mottling of the fundus occurs. This was less striking in the heavily pigmented fundus of a Eurasian patient.

Pallor around the Disc.-Obvious changes in the fundus colour, particularly between the disc and the macula, occur within 15 to 17 minutes of occlusion.

Peripheral pallor.-This has been observed within from 17 to 21 minutes of occlusion but is not a constant finding. 
Retinal Vessels Invisible, Marked Extension of Other Signs.-Within from 20 to 22 minutes of occlusion, the changes previously noted become more marked and the retinal vessels become very difficult to see. The veins are still more prominent than the arteries, which in many cases are quite invisible. At this stage, the retina is becoming pale and mottled. The disc is deadly white and shows no vessels either arising from or entering it.

Further Changes.-After 22 to 24 minutes of circulatory arrest, marked changes occur in the fundal picture. The fundus becomes gradually more mottled and pale, the vessels become extremely attenuated and in many cases quite invisible, the disc is very white and there is marked pallor in the discmacular area, and the periphery is quite white. In some cases, the macula has presented a dull greenish hue surrounded by a dark-grey area.

\section{BYPASS RE-WARMING}

On the return of arterial circulation through the bypass pump, there is immediate restoration of the intra-ocular circulation within 2 seconds. This has now been observed on many occasions and is most dramatic. Within 2 seconds the face flushes and at the same time there is a rush of blood into the arterial retinal circulation, with immediate restoration of the calibre of the arteries and veins and of the general fundus colour. This occurs so quickly that it is not possible to note any time-lag between the restoration of arterial and venous calibre. During the period after the restoration of circulation, first by bypass pumping and then via normal cardiac circulation, no change has been evident in the fundal picture. In particular, haemorrhage has been looked for but has not been seen, although subsequently found post-operatively.

Arterial Spasm.-Transitory spasm has been observed in two cases immediately following restoration of circulation.

\section{Post-operative Changes}

It has been considered clinically unwise to disturb these patients within 24 hours of the operation, and the first fundal examinations are therefore carried out on the second day.

Four cases have shown retinal transudates mainly near the macula and between the disc and the macula. One case, occluded for 31 minutes at $17^{\circ} \mathrm{C}$. $\left(11^{\circ} \mathrm{C}\right.$.), showed three small haemorrhages.

The transudates have been ill-defined and greyish at first, becoming whiter and more "cotton-wool" in appearance on the 5th or 6th day and then slowly regressing over a period of weeks.

In the one case so far observed with haemorrhages, complete resolution 
has taken place, and other cases which have shown post-operative petechial skin changes have not had retinal haemorrhages.

No patient has suffered visual damage.

Lacrimal Secretion.-Atropine premedication influences this study. The eyes remain moist during anaesthesia but become drier as the temperature falls. During circulatory arrest there is no secretion (Schirmer) and the cornea feels dry and "sticky" to touch. On re-circulation and re-warming, the tear production gradually returns but it has not yet been found possible to assess this accurately.

Cornea.-Hypothermia does not influence the corneal transparency. Some minutes after occlusion, however, a slow hazing of the epithelium has occurred in a few cases.

Refraction.-A change does occur in the refraction of the eye during these procedures. There is a quite marked increase in hypermetropia, up to +3 dioptres, which is presumably caused by the decrease in the anteroposterior axis of the eye due to the hypotension.

\section{PuPILS}

\section{Discussion}

The pupils behave basically according to expectation with the following exceptions:-

(1) The rapid mydriasis which occurs when the bypass cooling system is introduced is not clearly explained. It has been suggested that there is a pupillary dilator mechanism, associated with blood loss and vessel constriction (Davson, 1949).

It seems more likely that the rapid mydriasis is due to a hypothalamic disturbance with a degree of IIIrd nerve nuclear paralysis, arising from the fact that when the bypass cooling system is turned on there is a large influx into the arterial system of blood which is at a markedly lower temperature than the patient's blood.

That the mydriasis is certainly central in origin is proved by the experiments with local miosis.

(2) The miotic pupil obviously remains under the influence of the miotic during hypothermia and occlusion. This implies that the constrictor muscle of the pupil is still retaining some degree of tone.

Two other pupillary reactions should be noted:

(a) In dogs electrical defibrillation produces a markedly delayed (one second) pupillary enlargement with a slow return to normal. A second shock occurring during the active pupillary phase produces a summation resulting in maximum mydriasis with a slow- 45 seconds-return to normal.

(b) In man the pupil response is minimal. 
The electrical stimulus may therefore be assumed to act relatively to the body mass.

\section{InTra-ocular Pressure}

Hypothermia per se does not produce a fall in intra-ocular pressure.

Circulatory arrest results in profound hypotension but does not act immediately, the shortest time so far observed being 16 seconds.

During occlusion the blood within the eye, both retinal and choroidal, gradually drains away, possibly into the larger venous channels, and this is accompanied by a further hypotensive response.

When circulation is restored, the blood pressure immediately recovers to a mean of $50 \mathrm{~mm}$. $\mathrm{Hg}$, but the intra-ocular pressure has not been found to start recovery for 15 seconds-in dogs 7 seconds (Fig. 5).

Thereafter the increase in both blood and intra-ocular pressure bears a fairly close relationship which is not reflected in the body temperature increase.

\section{Fundus Changes}

The complete arrest of circulation in a human body almost ice-cold to touch $\left(10^{\circ} \mathrm{C}\right.$. thoracic) is a dramatic event. The ancient concepts of life and death become very real. To place a hand on the cold forehead and look through the slightly misty cornea and wide, dilated pupil at the gradual death of the fundus is a strange experience. To give these changes a scientific explanation is not easy, but they fall under four main headings:

(1) The sudden disappearance of arterial pressure (flow) results in the collapse of the smaller vessels with immediate disc pallor followed by a merging of the arterial and venous blood to a uniform bluish colour.

(2) Gravitational drainage leads to fragmentation of the blood columns with collapse of the vessels where they cross the disc margin.

(3) Gradual anoxic and hypotonic changes, characterized by loss of blood volume through the vessel walls, result in changes in the neighbouring tissues.

(4) Further tissue deterioration occurs which can only be described as post mortem in type.

During the present observations it has seemed evident that these changes occur in proportion to the degree of hypothermia in relation to the length of the occlusion. At present, with hypothermia of $16^{\circ} \mathrm{C} .\left(10^{\circ} \mathrm{C}\right.$.), 23 minutes of occlusion had not been followed by any post-operative fundus changes. Occlusion extended beyond this time may be expected to result in some degree of visible organic retinal damage post-operatively. 
In view of the drainage of blood from the eye, therefore, and possibly from the brain during occlusion, it is suggested that it might be advisable to lower the head to lessen the degree of anoxia, but there may be objections to this on the grounds that increased venous congestion might lead to postoperative cerebral oedema.

The gravitational drainage from the retina suggested that it might be wise to leave the large venous reservoirs of the body full. This was done in our most recent operation, in which the circulation was occluded for 43 minutes at $10^{\circ} \mathrm{C}$. (thoracic). The retinal vessels remained much more full of blood than usual, the post-operative retinal changes were minimal and the patient progressed very well generally.

The sudden restoration of blood pressure in the arterioles of the retina when the intra-ocular pressure is nil might possibly cause haemorrhage. On the other hand, this does not occur in the sudden reduction in intraocular pressure which takes place in operations involving the sudden opening of the eye such as cataract extraction, etc.

It seems, therefore, that the pathological changes observed in these cases may be due not so much to the sudden pressure rise in these vessels, but to the fact that they were not able to stand this rush of blood because of damage to their structure. The changes are very similar to those occurring in other retinopathies of metabolic origin in which the endothelial lining of the capillaries has been damaged. Arising from this it would seem that anoxia is the basic cause for these pathological changes.

Should post-operative organic changes occur at the macula, then visual damage of a serious nature could occur which might prove irreversible.

The possible effects of the hypothermia, anoxia, and electrolytic changes on the lens have been considered and investigations in that direction have been started.

The similarity of the retinal and cerebral blood-supply has been kept in mind. The retinal arteries are essentially arterioles in that they have no muscular coat, and it has also been suggested that they have their own local blood pressure regulator mechanism. This should be remembered when the question of the retinal changes is considered in relation to possible concurrent cerebral changes. In other words, it does not follow that, because retinal changes have been observed, cerebral damage must have occurred. This has been borne out clinically in our cases.

The possibility of haematuria has been investigated because of the close anatomical similarity between the retinal arterioles and those of the renal glomeruli, but the results of these observations were negative.

On the other hand, it should be realized that, should arterial or other retinal disease be present pre-operatively, then the question of operation in such cases might have to be carefully reviewed from the point of view of damage to the patient's eyesight. So far the patients have been young, 
and it is anticipated that the use of the present technique will be mainly confined to young people.

Nevertheless, its use may be extended in the future, and if this is so more elderly people may be subjected to this form of hypothermic occlusion operation. It is therefore very important that all patients who are to be treated by this procedure should have very careful ocular surveys both before and after operation.

\section{SUMMARY}

A new technique of profound hypothermia with total circulatory arrest for up to 43 minutes to facilitate intracardiac surgery is described.

The first observations on the ocular changes are reported.

Particular note is made of the behaviour of the pupil, ocular tension, fundus picture, cornea, and lacrimal secretion. The importance of postoperative fundus changes in relation to the possibility of brain and other tissue damage is discussed.

I wish to express my thanks to Mr. J. A. Simpson and the members of the Cardiac and Thoracic Surgical Units of the Royal Perth Hospital and Princess Margaret Hospital and to Mr. R. Plummer, F.R.P.S., Head of Medical Photography, Public Health Department, Government of Western Australia.

\section{REFERENCES}

Davson, H. (1949). “"The Physiology of the Eye", p. 237. Churchill, London. DREW, C. E., and ANDERSON, I. M. (1959). Lancet, 1, 748.

Simpson, J. A., Gibson, P., and Bloomfield, D. A. (1960). Med. J. Aust., 1, 647. 